\title{
ISCEV Standard for clinical electro-oculography (2017 update)
}

\author{
Paul A. Constable • Michael Bach · Laura J. Frishman • Brett G. Jeffrey • Anthony G. Robson • \\ for the International Society for Clinical Electrophysiology of Vision
}

Received: 9 January 2017/ Accepted: 11 January 2017 / Published online: 21 January 2017

(C) Springer-Verlag Berlin Heidelberg 2017

\begin{abstract}
The clinical electro-oculogram (EOG) is an electrophysiological test of the outer retina and retinal pigment epithelium (RPE) in which changes in the electrical potential across the RPE are recorded during successive periods of dark and light adaptation. This document presents the 2017 EOG Standard from the International Society for Clinical Electrophysiology of Vision (ISCEV: www.iscev.org). This standard has been reorganized and updated to include an explanation of the mechanism of the EOG, but without substantive changes to the testing protocol from the previous version published in 2011. It describes methods for recording the EOG in clinical applications and gives detailed guidance on technical requirements,
\end{abstract}

This update was approved by ISCEV. This document is available on the ISCEV website: http://www.iscev.org.

The authors represent the International Society for Clinical Electrophysiology of Vision.

\section{P. A. Constable $(\square)$}

Discipline of Optometry and Vision Science, Flinders

University, Adelaide, Australia

e-mail: paul.constable@flinders.edu.au

\section{Bach}

Department of Ophthalmology, University Medical

Center Freiburg, Freiburg, Germany

\section{J. Frishman}

College of Optometry, University of Houston, Houston,

TX, USA practical issues and reporting of results with the main clinical measure (the Arden ratio) now termed the light peak:dark trough ratio. The standard is intended to promote consistent quality of testing and reporting within and between clinical centers.

Keywords ISCEV Standards · Clinical electrophysiology · Electro-oculogram (EOG) · Arden ratio $\cdot$ Light peak:dark trough ratio $\cdot$ Light adaptation . Retinal pigment epithelium (RPE) · Fast oscillation (FO)

$\begin{array}{ll}\text { Abbreviations } \\ \text { DT } & \text { Dark trough } \\ \text { EOG } & \text { Electro-oculogram } \\ \text { ER } & \text { Endoplasmic reticulum } \\ \text { ERG } & \text { Electroretinogram } \\ \text { FO } & \text { Fast oscillation }\end{array}$

B. G. Jeffrey Ophthalmic Genetics and Visual Function Branch, National Eye Institute, Bethesda, MD, USA

A. G. Robson Institute of Ophthalmology, University College London, London, UK

A. G. Robson Moorfields Eye Hospital, London, UK 


$\begin{array}{ll}\text { ISCEV } & \begin{array}{l}\text { International Society for Clinical } \\ \text { Electrophysiology of Vision }\end{array} \\ \text { LP } & \text { Light peak } \\ \text { LP:DT } & \text { Light peak:dark trough ratio } \\ \text { ratio } & \\ \text { RPE } & \text { Retinal pigment epithelium } \\ \text { TEP } & \text { Transepithelial potential }\end{array}$

This Standard is one of the series of ISCEV Standards and Guidelines for clinical electrophysiology of vision [1-7]. Current standards are listed on the ISCEV Web site [8]. This Standard supersedes previous versions of the ISCEV Standard for clinical electro-oculography which was first published in 1993 [1] and subsequently revised most recently in 2011 [2]. This Standard contains updated information on the mechanism and reporting of the EOG and has been reorganized for greater clarity and for consistency with other ISCEV Standards, without substantive changes to the 2011 testing protocol.

This ISCEV Standard describes basic procedures that allow reproducible recordings that are comparable across laboratories. It is intended that the ISCEV Standard EOG protocol be used widely, but not to the exclusion of other tests or protocols that are not covered by this Standard. Electrophysiologists are encouraged to extend the EOG as required when clinically relevant to maximize the diagnostic value of the EOG and fast oscillation (FO) recordings.

Clinical and research users of the EOG are encouraged to use the most recent Standard, to achieve consistency of results within and between test centers. Reports of EOG recordings performed in this manner should cite this 2017 Standard. When a method is used which deviates from the Standard, the deviations should be fully described.

\section{The electro-oculogram}

Electrophysiology of the outer retina in dark and light

There is a difference in electrical potential between the anterior and posterior of the eye, known as the standing potential of the eye. The standing potential is an indirect measure of the transepithelial potential
(TEP) of the retinal pigment epithelium (RPE). The TEP is equal to the difference in the membrane potential of the basolateral and the apical membranes, which are electrically isolated through the tight junctions of the RPE. Changes in the resistance between the apical and basolateral membranes or membrane potentials alter the amplitude of the TEP and thus affect the recorded standing potential of the eye. A change in the standing potential can be induced by retinal adaptation to a change in ambient illumination.

There are two clinical tests of the standing potential of the eye. The EOG utilizes the RPE's response to changing illumination to assess the function of the outer retina and RPE. The EOG is recorded during $15 \mathrm{~min}$ of dark adaptation followed by $15 \mathrm{~min}$ of light adaptation. The FO is a different EOG procedure performed during alternate 1-min dark and light periods. The changes in the standing potential for the EOG and the FO are derived from different mechanisms.

\section{Mechanism of the EOG}

During the 15-min period of dark adaptation, there is a fall in the recorded standing potential typically reaching a minimum at $10-15 \mathrm{~min}$, and this is referred to as the dark trough (DT). Following light onset, there is an increase in the TEP of the RPE producing the light-rise of the EOG. The mechanism initiating the light-rise is unknown, but it requires a normally functioning interface between the rod photoreceptors and RPE. The light-rise is ultimately the result of an increase in intracellular free calcium, which is released from the endoplasmic reticulum (ER), regulated by an interaction between ER bestrophin and L-type calcium channels associated with the basolateral membrane. Intracellular calcium gates the opening of a basolateral calcium-dependent chloride channel. Increased chloride conductance depolarizes the basolateral membrane which increases the TEP, recorded as an increase in the standing potential of the eye. The light-rise normally reaches a maximum at 7-12 min after light onset and is known as the light peak (LP). The LP is the first of several peaks (the damped oscillation) which become progressively smaller for up to $90 \mathrm{~min}$ during continued light exposure. 
The clinical EOG provides an indirect measure of the minimum amplitude of the standing potential in the dark (at the DT) and then again at its maximum amplitude in the light (at the LP). This is expressed as the EOG light peak-to-dark trough ratio (LP:DT ratio). ISCEV recommends the term LP:DT ratio when reporting this result.

\section{Mechanism of the FO}

The FO is an optional additional test that has a different mechanism to the clinical EOG owing to the shorter dark and light intervals used. At light onset, there is a fall in potassium in the sub-retinal space that causes a strong outward hyperpolarizing potassium current across the RPE's apical membrane and is reflected in the c-wave of the electroretinogram (ERG). The fall in sub-retinal potassium also reduces the transport of chloride ions into the RPE. The reduction in chloride ions causes the basolateral membrane to hyperpolarize and lowers the TEP generating the trough of the FO 35-45 s after light onset. The TEP returns to normal, as ionic homeostasis is restored and a peak is recorded during the subsequent dark period after a further $35-45 \mathrm{~s}$. The alternation between dark and light at 1-min intervals establishes a continuous oscillation that is dependent on changes in ionic permeability at the apical and basal membranes and the electrical coupling between these membranes by tight junctions.

\section{Diseases affecting the light response of the EOG}

The LP:DT ratio of the EOG is affected in some diffuse disorders of the RPE and disorders of the photoreceptor layer of the retina including acquired retinopathies and retinal dystrophies characterized by rod dysfunction or chorio-retinal atrophy. In most of these disorders, EOG abnormalities are proportional to the severity of rodmediated ERG abnormalities and are not of diagnostic importance. Notable exceptions include disorders of the bestrophin gene (BEST1). These include Best vitelliform macular dystrophy (Best disease), autosomal recessive bestrophinopathy (ARB) and autosomal dominant vitreoretinochoroidopathy (ADVIRC). In Best disease standard, full-field ERGs are usually normal and the LP:DT ratio of the EOG abnormal. An abnormal EOG may distinguish Best disease from other autosomal dominant retinal disorders with similar fundus features including some cases of adult vitelliform macular dystrophy and pattern dystrophy. In ARB and ADVIRC, the ERG is often abnormal but the EOG is severely or disproportionately abnormal. An abnormal EOG, not explained by ERG reduction, may also be associated with some toxic retinopathies.

\section{Principles of the clinical EOG measurement}

The standing potential of the eye may be assessed using skin electrodes placed near the outer and inner canthi of each eye to record successive horizontal saccadic eye movements. The patient tracks alternating lights separated by a fixed angle, to enable constant eye movement excursions which are recorded as a series of positive and negative deflections that coincide with ocular rotation. The magnitude of the eye movement potential (at a fixed angle) is proportional to the standing potential.

\section{The standard method}

Technologic requirements

\section{Electrodes}

Skin electrodes such as sintered silver-silver chloride, standard silver-silver chloride or gold cup electrodes are recommended for recording the EOG. The skin should be prepared by cleaning, and a suitable paste or gel used to ensure good, stable electrical connection. The electrode-skin contact impedances should be below $5 \mathrm{k} \Omega$ as measured between 20 and $40 \mathrm{~Hz}$.

\section{Stimulator}

Full-field (ganzfeld) stimulation must be used to provide uniform luminance over the entire visual field of the patient. This is usually achieved using a dome or integrating sphere. It is incumbent on manufacturers and users to verify that stimulation meets the full-field requirement of this Standard and provides a comfortable head/chin rest for the patient. Two red fixation lights located $15^{\circ}$ left and right of center should be bright enough to be just visible to the patient during the dark and light phases of the recordings. 


\section{Light and dark}

The adapting dark phase should take place in total darkness, with the fixation lights dimmed to the minimum necessary to enable fixation for the patient.

The adapting light phase requires a white light with a luminance of 100 photopic $\mathrm{cd} \mathrm{m}^{-2}$ measured at the position of the eye. To account for minor variability in equipment and calibration, the acceptable range within the Standard for the light adapting background is $90-110$ photopic $\mathrm{cd} \mathrm{m}^{-2}$. Calibration of the ganzfeld stimulator should be carried out periodically. Modest room lighting may be turned on during the light phase providing the ambient luminance is less than that in the ganzfeld.

Note that adapting light sources of different types, such as tungsten, halogen, LED and fluorescent bulbs, have different spectral characteristics and the color may change with brightness. This makes the definition of standard lighting inherently imprecise, although for practical purposes "white" light of the correct luminance will give similar results.

\section{Amplification}

Amplifiers should have a band pass of either DC to 30 or $0.1-30 \mathrm{~Hz}$ to make recordings of saccadic eye movements appear as square waves (Fig. 1). For a $30^{\circ}$ saccade, the typical EOG amplitude is between 250 and $1000 \mu \mathrm{V}$ with an essential frequency content of 0-30 Hz. To avoid a loss of information, digitizers should sample the saccades at a rate of $1 \mathrm{kHz}$ or higher in each channel. In theory, the ideal recording technique is DC amplification but this is generally impractical because of baseline drift. Thus, we recommend $\mathrm{AC}$ coupling with a $0.1 \mathrm{~Hz}$ high-pass and $30 \mathrm{~Hz}$ low-pass filter. If a higher-frequency filter is used (e.g., $0.5 \mathrm{~Hz}$ ), it will distort the square waves, making identification of overshoot and stepped saccades difficult.

Manufacturers should allow the examiner to have access to all of the raw data for each 10-s recording epoch so that the examiner is able to visually inspect the individual saccadic records to enable accurate cursor placement around any artifacts to measure the standing potential as shown in Fig. 2.

The operator should be able to visually inspect saccadic recordings as they are performed to monitor for artifacts including those caused by blinks, inconsistent eye movements, patient compliance problems, poor electrode contact or amplifier saturation. Prompt detection allows artifacts to be minimized or eliminated, e.g., by encouragement of the patient, reapplication of recording electrodes or by making necessary adjustments to the amplifier gain.

Preparation of the patient

\section{Pupils}

The pupils should be dilated before testing and their size or diameter recorded. If full pupil dilation is impossible or undesirable, an attempt should be made to increase the adapting luminance so that an equivalent retinal illumination is approximated. The amount of light passing through the pupil, when measured in Trolands, is the product of luminance (in $\mathrm{cd} \mathrm{m}^{-2}$ ) and pupil area (in $\mathrm{mm}^{2}$ ). For example, to produce the same effect upon the retina, twice as much luminance is required with a 5-mm diameter pupil (roughly $20 \mathrm{~mm}^{2}$ ) than with a 7-mm diameter pupil (roughly $40 \mathrm{~mm}^{2}$ ). The report should describe any deviations from the Standard.

\section{Electrode placement}

After suitable skin preparation, recording electrodes should be placed close to the canthi of each eye as in Fig. 3. The electrodes from each eye are connected to separate channels of a differential amplifier. A separate electrode should be attached and connected to the ground. Convenient and commonly used ground electrode positions include the forehead, vertex, mastoid and earlobe. The impedance between any pair of electrodes should not exceed $5 \mathrm{k} \Omega$. The electrodes, amplifier and impedance meter must be approved for medical use.

\section{Pre-exposure to light}

The patient should be in stable indoor lighting for at least $30 \mathrm{~min}$ before the test. Indirect ophthalmoscopy, fundus autofluorescence, fundus photography and other strong illumination changes must be avoided during this period. As near as practical, the pretest light exposure should be the same for all patients. 

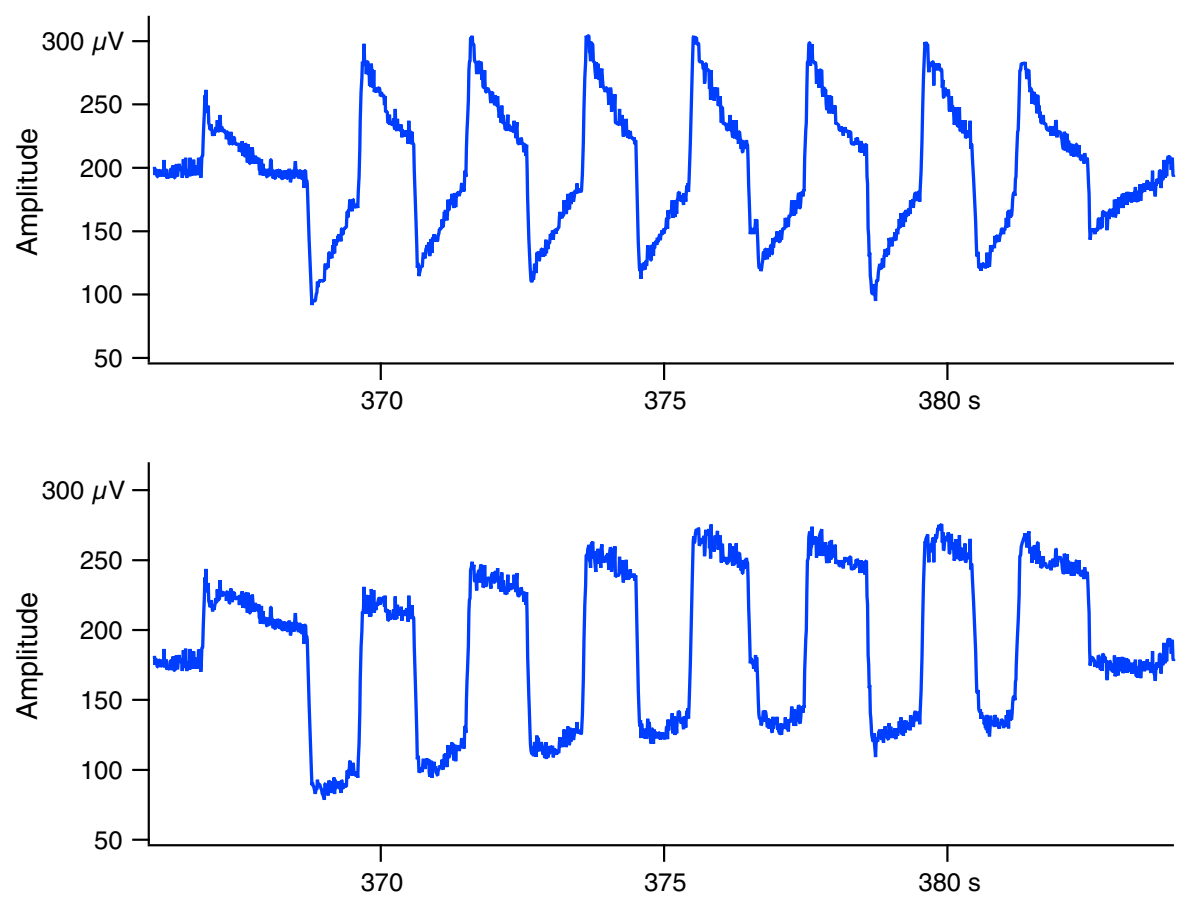

Fig. 1 Upper trace shows filtering with $0.1-30 \mathrm{~Hz}$, lower trace with post hoc DC restoration by digital integration, rendering it similar to direct DC recording. DC recording (or restoration) makes it easier to perform the plateau measurements

Fig. 2 Examples of 10-s saccadic records with a blink artifact at approximately $4000 \mathrm{~ms}$. Arrow a indicates an initial undershoot and arrow $b$ an overshoot of the fixation target visible by the step in the plateau of the EOG recording (upper trace). The lower trace shows the manual placing of markers at the peak and trough of the EOG recording as the eye performs horizontal saccades for $10 \mathrm{~s}$ at 1-s intervals
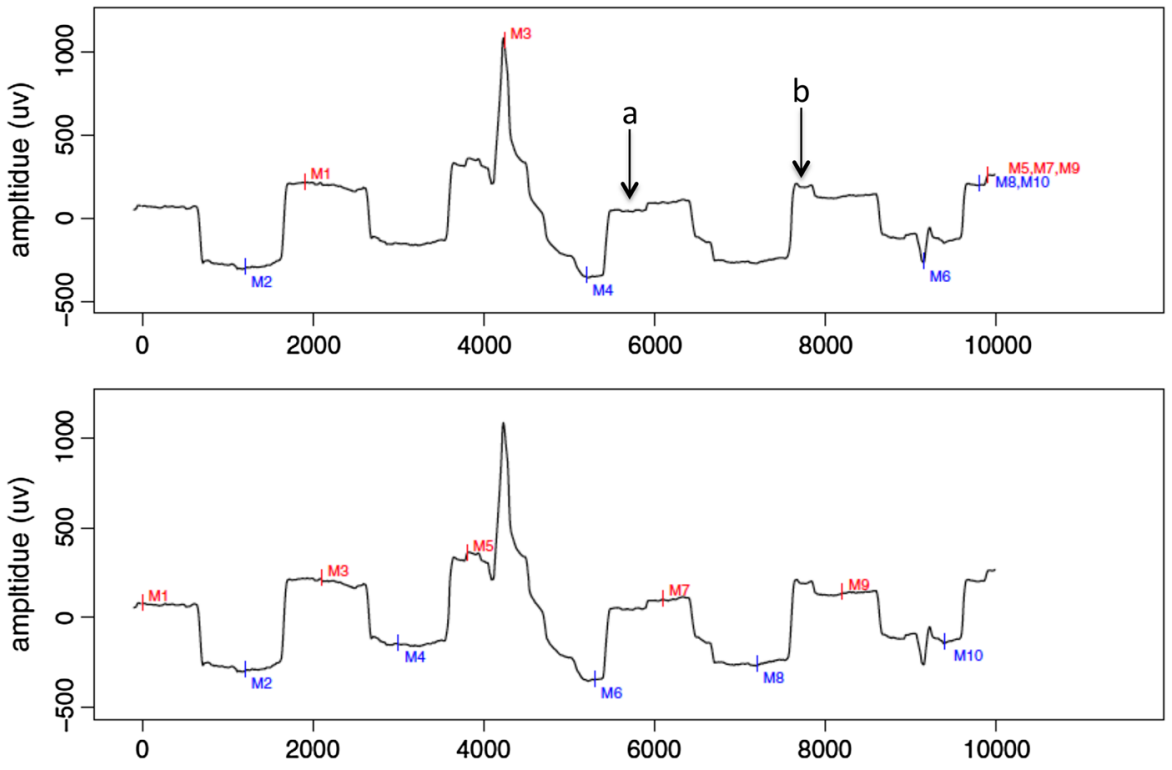

\section{Instructions to the patient}

The procedure should be explained to the patient, noting that head position must not change, as this is one common source of artifact and that the eyes should only move left and right. Patients should be instructed not to anticipate the onset of the alternation of the fixation lights but to move their eyes only when the lights change. Practice the procedure with the recording system prior to dark adaptation, to familiarize the patient with the task and to check on the stability and quality of the recorded saccades. 

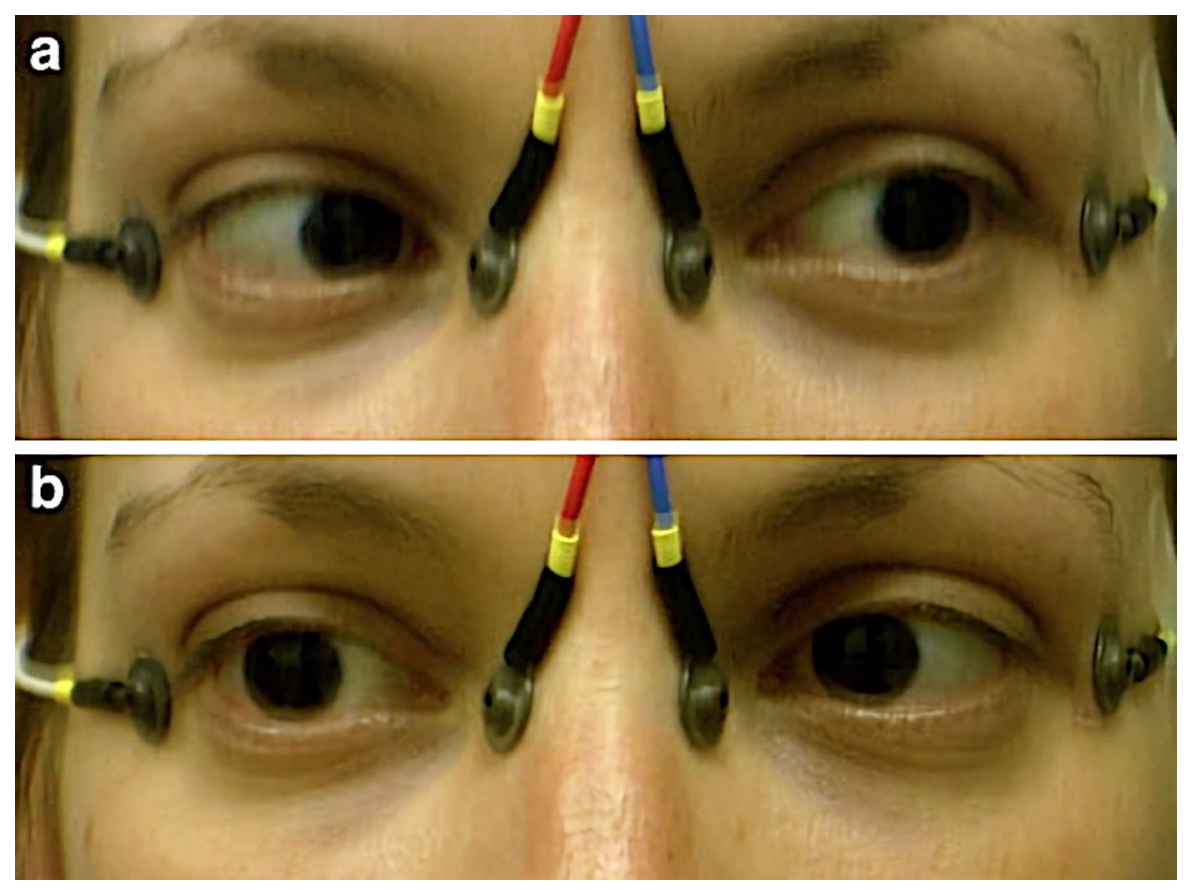

Fig. 3 Recording electrode positions located near the inner and outer canthi of each eye. As the eyes perform horizontal saccades: left (a) then right (b), the amplitude of the standing potential is recorded across the active electrodes

\section{Clinical recording}

\section{Recording of saccades}

Fixation lights should alternate once per second, for $10 \mathrm{~s}$ out of every minute. The EOG potentials are recorded during each of these 10-s periods. There should be a warning, verbal or automatic, of the impending start of each recording period to ensure readiness of the patient and operator. Auditory cues during the recordings may be used and can be helpful in patients with restricted visual fields.

\section{Dark phase}

For the dark phase, total darkness should be maintained for $15 \mathrm{~min}$, except for the dim fixation lights. EOG recordings should be made once a minute for $10 \mathrm{~s}$, as specified above. The operator should have a concurrent view of the recordings to check for patient compliance and for errors such as noise or overshoot.

\section{Light phase}

For the light phase, a ganzfeld background light of 100 photopic $\mathrm{cd} \mathrm{m}^{-2}$ should be turned on to initiate the light phase response and should remain on for the duration of the light phase. However, the luminance can be increased gradually over a short period (e.g., $20 \mathrm{~s})$ to minimize patient discomfort. Longer ramps (e.g., lasting minutes) will alter the responses. Continue the recording for $10 \mathrm{~s}$ out of every minute (as above) for at least $15 \mathrm{~min}$ to register the presence or absence of the LP. If the LP can be clearly identified during the recordings, then the test may be stopped before $15 \mathrm{~min}$. It may be necessary to extend the light phase to fully characterize abnormal responses, but a delay in the LP should be reported. The patient should remain positioned in the headrest of the stimulator throughout the procedure, with eyes open to maintain retinal illumination.

\section{Patient compliance}

Patients will have difficulty performing saccadic eye movements if they cannot fixate reliably because of poor central vision, diplopia or ocular motility problems (including nystagmus). Patients with diplopia may be advised to look between the pair of images, or one eye can be patched if the suspected retinal disorder is binocular. Patients who are very young or those with 
a physical or learning disability may not be able to perform the EOG. In young children with suspected Best disease, it may be useful to test their parents, since a carrier of Best disease will have an abnormal light-rise, irrespective of whether the fundus is normal.

Patient compliance can vary due to fatigue or inattention. Common problems include gradual movement of the head back from the stimulator, head turning, irregular eye movements during the recording or eye closure during the light phase can be minimized by having a real-time view of the patient's eyes via an infrared camera. In most cases, gentle coaching and reminders will minimize the effects of poor compliance.

\section{Analysis and reporting}

\section{Saccadic amplitude}

The EOG amplitudes should be measured in microvolts $(\mu \mathrm{V})$ either manually or by a computer algorithm after visual inspection. Care must be taken to avoid measuring the effects of overshoot (see Fig. 2) or irregular (artifactual) saccades. The average of the amplitudes within each 10-s recording epoch should be measured, excluding outliers, artifacts or responses consistent with poor eye movement accuracy. If a computer algorithm is used, it is important to ensure that the values obtained represent true EOG amplitudes and not artifactual records or outliers. Common causes of unreliability are overshoot of the fixation lights, stepped rather than smooth saccades, missing saccades, inverse saccades (eyes going in the opposite way to the fixation lights), and eccentric fixation in which the saccade length switches between two or more values.

\section{Dark trough and light peak: smoothing}

The average EOG amplitude calculated from each 10 -s epoch should be plotted. However, there is always "noise" in physiological recordings, and the goal of the EOG measurement is to record the underlying DT and LP, rather than the lowest or highest single values. Thus, the first critical step is that the underlying physiologic curve be recognized and drawn, in order to derive reliable DT and LP amplitudes. This can be achieved by various methods including visual inspection, by use of a flexible spline rule, computer-based curve fitting algorithms or weighted averages of the EOG amplitudes.

Figure 4 shows raw data plotted and the subsequent smoothing of the data points using, in this case, the weighted mean of the recorded EOG amplitudes at each time point. It is helpful if uncertain or artifactual values can be identified and marked at the time of recording, so that they can be ignored later when smoothing and curve fitting.

\section{Light peak-to-dark trough ratio calculation}

The LP:DT ratio of the EOG is computed by dividing the smoothed LP by the DT amplitudes. It is important to note that a normal LP:DT ratio does not imply a normal DT and this amplitude should be reported as a measure of the standing potential.

\section{Reporting}

Clinical reports should state the EOG LP:DT ratio, the DT amplitude (in microvolts) and the time from the start of the light phase to the LP (if present). The type of adapting light source and pupil size should also be reported. The report should also describe any difficulties encountered during testing that may affect confidence in the results, such as patient compliance or inconsistent eye movements.

\section{Normative data}

Each center must establish its own set of normative values for the EOG and FO. The median value (not the mean) should be used to define reference values and the actual values on either side of the median that bracket 90 percent of the reference ranges (5th-95th centile) should be determined by direct tabulation of the reported results. The normal LP:DT ratio is typically between 1.7 and 4.3 with a LP time ranging from 7 to $12 \mathrm{~min}$.

\section{Interaction between eyes}

The EOG potentials from one eye can contaminate the response from the other. The magnitude of this effect is approximately $15 \%$ with electrodes placed on each side of the nose close to the inner canthi. It rises to about $40 \%$ if the electrodes come close together and 
Fig. 4 Upper figure shows the raw standing potential values for an EOG with 15 min of dark (black circles) and 15 min of light (white circles). Smoothing of the data helps to define the DT and LP amplitudes from which to calculate the light peak:dark trough ratio (lower figure). Computer algorithms or fitting with a spline rule may also be utilized
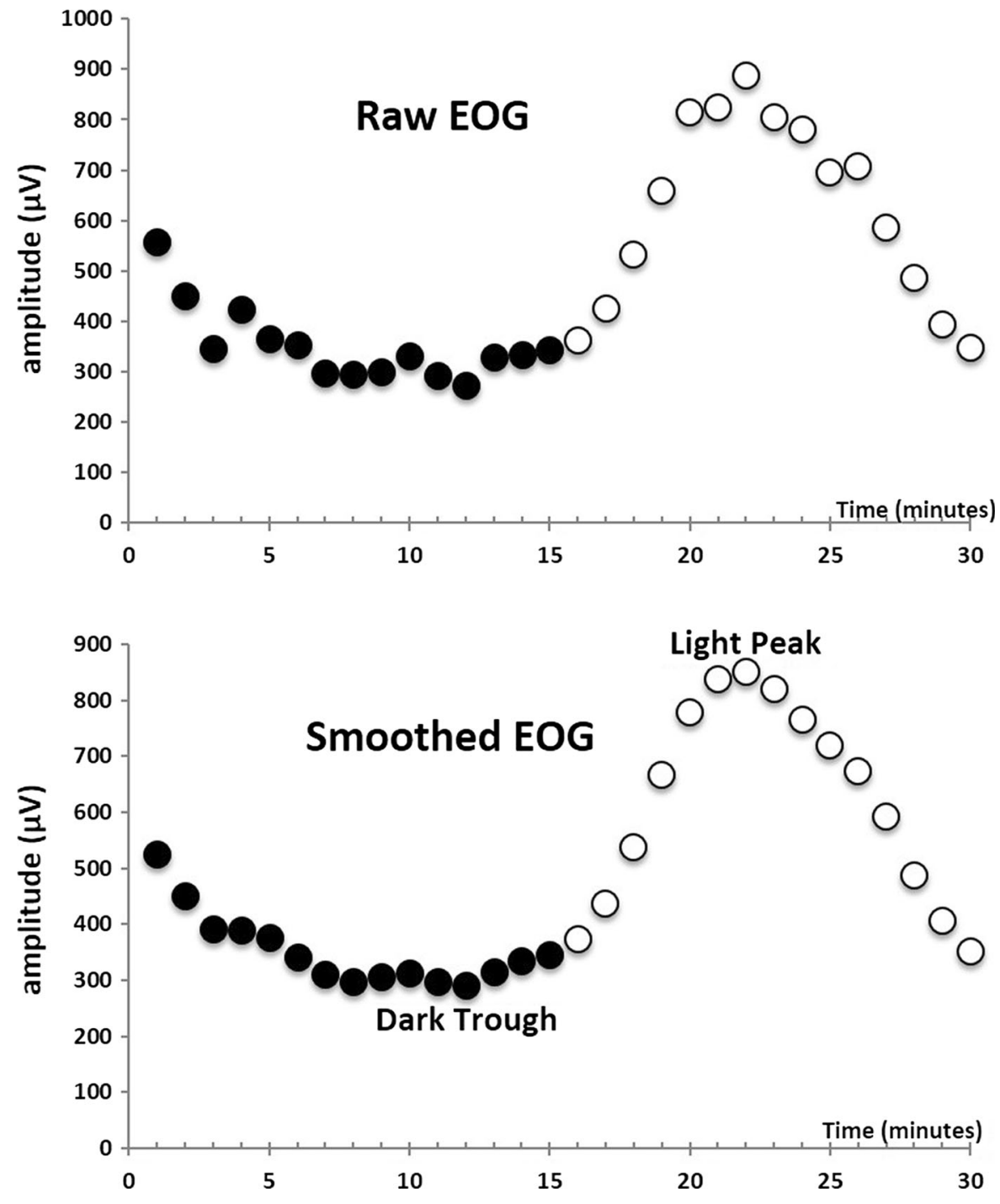

touch, becoming equivalent to a common central electrode on the bridge of the nose. This interaction can give misleading results if an eye is electrically inactive, e.g., in total retinal detachment or absent. This is because the defective eye will appear to have the same LP:DT ratio as the fellow eye, but with a much smaller standing potential (DT amplitude). If the eyes have similar standing potentials but a different LP:DT ratio, then the measured ratio from the better eye is enhanced at the expense of that from the weaker eye.

\section{Deviation from the Standard}

This Standard represents a basic and core procedure for the assessment of generalized function of the RPE/ photoreceptor interface. If a center chooses techniques that vary from the Standard, such as differences in the luminance level for the adapting light or in the duration of the dark or light adapting intervals, it is critical to cite this document and specify any deviations. The standing potential of the EOG can also be used to monitor eye movements in studies unrelated to retinal and RPE pathophysiology.

\section{Fast oscillation}

The FO has the opposite polarity to the EOG. Light causes a decrease in the standing potential, while in darkness there is an increase in the standing potential. 


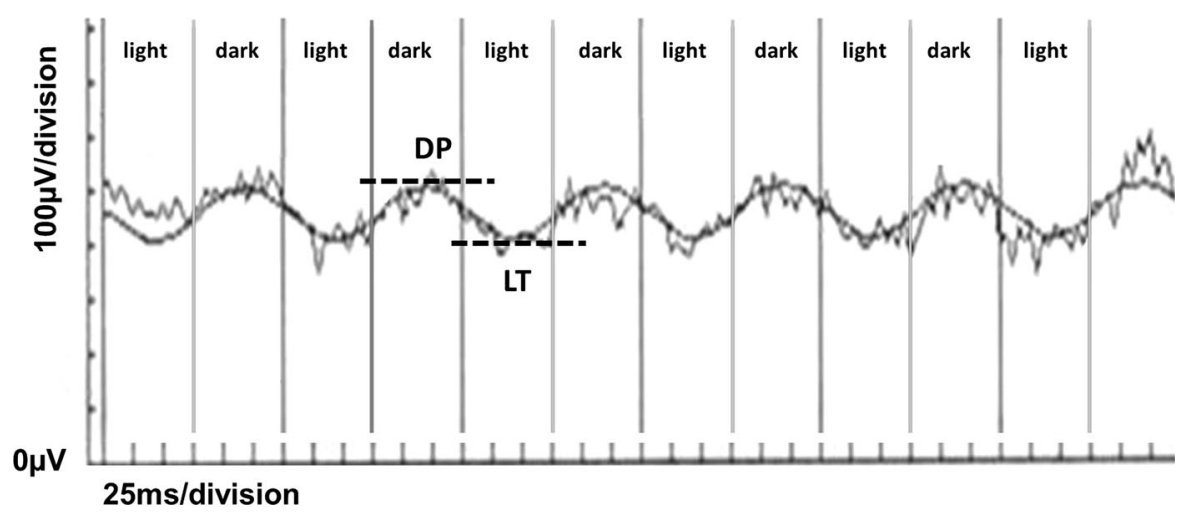

Fig. 5 Typical fast oscillation recording from a normal eye with six cycles of light and dark intervals (75 s each). There is a light trough (LT) and a dark peak (DP). In this case, amplitude

The FO is recorded using the same technical specifications as the EOG (amplifier, electrode placement, fixation targets, background luminance and $1 / \mathrm{s}$ saccades). However, the saccades and the recording should be continuous for the duration of the test. Light and dark intervals are alternated at 60 or 75 -s intervals to induce the FO, which has a near sinusoidal appearance (Fig. 5). The total number of light-dark intervals should be at least 4 . Pre-adaptation does not affect the FO, so this test can be performed either independently or before the EOG.

Figure 5 shows a FO recording formed by dark and light intervals. The FO amplitude can be calculated from the average of the peak-to-trough ratios from each of the dark-light cycles. The time to the peak or trough should be calculated from the time of light offset or onset, respectively, and averaged for the number of light-dark cycles in the recording.

Acknowledgements The ISCEV Standardization process depends on active participation of individual ISCEV members who act as consultants to the committee and who vote to approve the final document. We thank Mitchell Brigell, Michael Marmor and Daphne McCulloch in particular for their constructive input into this document.

\section{Compliance with ethical standards}

Conflict of interest The authors declare no conflict of interests.

Statement of human rights This article does not contain any studies with human participants performed by any of the authors. measurements were derived from the fit of the sine wave to the raw data. The FO ratio is computed from the amplitude of the DP divided by the amplitude of the LT (1.3)

Statement on the welfare of animals This article does not contain any studies with animals performed by any of the authors.

Informed consent As this article does not contain any studies with human participants performed directly by any of the authors, the concept of informed consent is not applicable.

\section{References}

1. Marmor MF, Zrenner E (1993) Standard for clinical electrooculography. International Society of Clinical Electrophysiology of Vision. Doc Ophthalmol 85(2):115-124

2. Marmor MF, Brigell MG, McCulloch DL, Westall CA, Bach M (2011) ISCEV Standard for clinical electro-oculography (2010 update). Doc Ophthalmol 122(1):1-7

3. Brigell M, Bach M, Barber C, Moskowitz A, Robson J (2003) Guidelines for calibration of stimulus and recording parameters used in clinical electrophysiology of vision. Doc Ophthalmol 107(2):185-193

4. McCulloch DL, Marmor MF, Brigell MG, Hamilton R, Holder GE, Tzekov R, Bach M (2015) ISCEV Standard for full-field clinical electroretinography (2015 update). Doc Ophthalmol 130(1):1-12

5. Hood DC, Bach M, Brigell M, Keating D, Kondo M, Lyons JS, Marmor MF, McCulloch DL, Palmowski-Wolfe AM (2012) ISCEV Standard for clinical multifocal electroretinography (2011 edition). Doc Ophthalmol 124(1):1-13

6. Bach M, Brigell MG, Hawlina M, Holder GE, Johnson MA, McCulloch DL, Meigen T, Viswanathan S (2013) ISCEV Standard for clinical pattern electroretinography (PERG) 2012 update. Doc Ophthalmol 126(1):1-7

7. Odom JV, Bach M, Brigell M, Holder GE, McCulloch DLL, Mizota A, Tormene AP (2016) ISCEV standard for clinical visual evoked potentials-(2016 update). Doc Ophthalmol 133(1):1-9

8. ISCEV Standards (2014) Retrieved 25 Oct 2016, from http:// www.iscev.org/standards/ 